\title{
Desórdenes informativos: sobreexpuestos e infrainformados en la era de la posverdad
}

\author{
Information disorders: Overexposed and under \\ informed in the post-truth era
}

\author{
Miguel Del-Fresno-García
}

Cómo citar este artículo:

Del-Fresno-García, Miguel (2019). “Desórdenes informativos: sobreexpuestos e infrainformados en la era de la posverdad". El profesional de la información, v. 28, n. 3, e280302.

https://doi.org/10.3145/epi.2019.may.02

Artículo recibido el 23 de abril de 2019 Aprobado el 8 de mayo de 2019

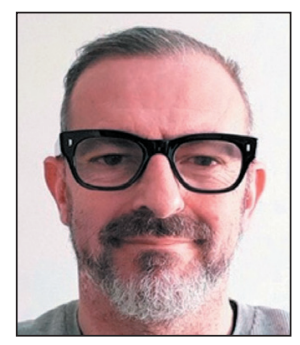

\section{Miguel Del-Fresno-García $\bowtie$ \\ https://orcid.org/0000-0003-2746-3681}

Universidad Nacional de Educación a Distancia (UNED)

Obispo Trejo, s/n. 28040 Madrid, España

mdelfresno@der.uned.es

\section{Resumen}

Los desórdenes informativos (desinformación, fake news, hechos alternativos, posverdad, deepfakes, etc.) son producciones intencionales cuya estrategia consiste en la fabricación de la duda y falsas controversias con el fin de conseguir beneficios económicos o ideológicos. Los desórdenes informativos están interrelacionados entre sí y dependen, de forma necesaria, de las tecnologías post Internet, lo que ha modificado la naturaleza misma de la comunicación interpersonal colectiva. Los desórdenes desinformativos tienen su origen y bases en distintas causas que han facilitado su desarrollo, alcance e impacto actual sin precedentes: a) la guerra contra la ciencia desde el ámbito corporativo, b) la crisis de los medios de comunicación nacionales y locales post Internet, c) el desarrollo de plataformas tecnológicas que han socializado la capacidad de publicar y distribuir contenidos a bajo coste, d) la crisis de los expertos con su consecuente crisis epistémica, e) los avances en psicología, para explotar las bases psicológicas de los desórdenes informativos, a través de diferentes sesgos cognitivos, y e) un cambio significativo en la forma de entender y ejercer el poder en el siglo XXI, como la capacidad de establecer las relaciones de definición (Beck, 2017) de la realidad misma. Los desórdenes informativos suponen una voluntad de autoridad sobre la realidad, en la práctica, una voluntad de supremacía ideológica, y un riesgo para las democracias liberales.

\section{Palabras clave}

Desórdenes informativos; Posverdad; Desinformación; Noticias falsas; Hechos alternativos; Deepfakes; Desinformación corporativa; Guerra a la ciencia.

\footnotetext{
Abstract

Information disorders (misinformation, fakes news, alternative facts, post-truth, deepfakes, etc.) are intentional productions whose strategy is focused on the fabrication of doubt and false controversies in order to achieve economic or ideological benefits. Information disorders are all interrelated and depend, in a necessary way, on post-Internet technologies which have modified the very nature of collective interpersonal communication. Information disorders have their origin and basis in different causes that have facilitated their development, scope and unprecedented current impact as: a) the war on science from the corporate sphere, b) the crisis of the post Internet national and local media, c) the development of technological platforms that have socialized the ability to publish and distribute content at low cost, d) the crisis of the experts with a consequent epistemic crisis, e) advances in psychology, that exploit the psychological bases of informative disorders through cognitive biases, and f) a significant change in the way of understanding and exercising
} 
power in the $21^{\text {st }}$ century, such as the ability to establish the relations of definition of reality itself (Beck, 2017). This supposes a true will of authority on the reality and, in the practice, a will of ideological supremacy and a serious risk for the liberal democracies.

\section{Keywords}

Information disorders; Post-truth; Misinformation; Fake news; Alternative facts; Deepfakes; Corporate missinformation; War on science.

\section{Introducción}

Lo que sucede con los conceptos ómnibus en la comunicación post internet es que acaban siendo utilizados como equivalentes y, en su proceso de extensión social se solapan significados y sentidos. Esto sucede con desinformación, fake news, hechos alternativos, posverdad, deepfakes, etc., como desórdenes informativos (Wardle; Hossein, 2017).

La producción de desórdenes informativos de forma intencional no es una novedad estricta de nuestro tiempo, por mucho que lo sean algunos conceptos, ya que la tecnología de cada época ha jugado un papel crítico a la hora de cambiar la escala e impacto de estos desórdenes en las sociedades. Varios pensadores han dejado importantes obras, por ejemplo, sobre la función política de la mentira (Koyré, 1945), su entramado con el totalitarismo (Arendt, 1951) o, más reciente, alrededor de nuestra incapacidad para diferenciar la verdad de la mentira (Frankfurt, 2005). Peter Hernon (1995) anticipó las posibilidades que ofrecía Internet para la alteración intencional de los significados al señalar que

"es fácil alterar el contenido de la información y pasar una falsificación como genuina" (Hernon, 1995, p. 134).

Paul Virilio $(1991,1995)$ ya había evidenciado como toda tecnología lleva implícito, en sí misma, su propio accidente y, debido a la misma evolución tecnológica, un creciente potencial catastrófico.

Los desórdenes informativos están interrelacionados entre sí y dependen de forma necesaria de las tecnologías post Internet, lo que ha modificado la naturaleza misma de la comunicación interpersonal colectiva. El interés científico en los desórdenes informativos post Internet reside en que no existen precedentes en su velocidad, alcance y distribución (Vosoughi et al., 2018; Del-Vicario et al., 2016) por medio de cascadas de información (Bikchandani et al., 1992). Tampoco hay precedentes en la forma y volumen de producción de los mismos ni en las consecuencias que provocan, o pueden provocar, de manera Los desórdenes informativos están interrelacionados entre sí y dependen, de forma necesaria, de las tecnologías post Internet lo que ha modificado la naturaleza misma de la comunicación interpersonal colectiva planetaria porque

"ahora el campo de batalla abarca toda la realidad factual [...] en un mundo en el que los políticos pueden desafiar los hechos y no pagar ningún precio político por ello" (Mclntyre, 2018, p. 43).

Esta metamorfosis se ha producido gracias a una

“compleja interacción entre prácticas comunicativas y sociales e infraestructura tecnológica” dentro de "un instantáneo y masivo sistema de comunicación en red" (Del-Fresno et al., 2016, p. 24)

lo que ha llevado a una nueva dimensión el riesgo de potenciales efectos catastróficos para la vida de millones de personas. Dos hechos sociopolíticos globales icónicos de las consecuencias de los desórdenes informativos fueron las elecciones presidenciales en EUA y el referéndum del Brexit en Reino Unido en 2016.

Verdad y mentira son conceptos filosóficos y, por tanto, sujetos a múltiples niveles de debate (ontológico, lógico, semántico, metafísico, ético, teológico, epistemológico, etc.). Tal como son presentadas aquí se refieren a verdades o mentiras fácticas. Esto es, la existencia o no de correspondencia entre enunciados y hechos. Cuando no existe tal correspondencia pero se sostiene de forma intencional que sí existe, estamos ante las fake news, que es conceptualmente lo más próximo a lo que el sentido común identifica como las mentiras de la propaganda. En todo caso, no estamos en un debate sobre la verdad o sus regímenes, en términos foucaultianos, sino sobre los desórdenes informativos, su identificación y sus consecuencias.

La desinformación tiene una mayor complejidad, en sí misma y para su identificación, que las fake news ya que para ser eficaz en su objetivo no necesita ser completamente falso. La desinformación consiste en la elección intencional de datos parciales, incompletos, alterados, etc. A falta de una definición aceptada de manera general, la desinformación es todo aquel contenido fabricado y distribuido

"falso, inexacto o engañoso [...] diseñado, presentado y promovido intencionalmente para causar daño público o beneficios" particulares (European Commission, 2018, p. 10) o "para engañar a las personas" (Lazer et al., 2018, p. 1094), 
o alterar la percepción de forma intencional de grandes grupos de personas o sociedades e influir en su comportamiento (político, económico, ideológico...). La negatividad de la desinformación se basa en la corrupción del proceso fiable de recogida y presentación de los hechos.

Así, los que la fabrican disponen de un extenso abanico de proposiciones híbridas, entre lo verdadero y lo falso, para generar la duda, el temor o la controversia con el objetivo de sesgar la percepción y el comportamiento de diferentes grupos sociales.

Fue a partir de 2016 cuando se socializó a escala mundial el concepto de posverdad como signo de una época (Drezner, 2016; Flood, 2016; Freedland, 2016; Alcorn, 2014). La definición más extendida de posverdad es la de aquellas

"circunstancias en las cuales los hechos objetivos son menos influyentes para moldear la opinión pública que apelar a la emoción y las creencias personales" (Oxford University Press, 2016).

Además, en relación con la capacidad de producción de efectos catastróficos, en

"su forma más pura la posverdad ocurre cuando alguien piensa que la reacción de las masas cambia realmente los hechos que son objeto de una mentira" (McIntyre, 2018, p. 38).

Esto es, la posverdad consiste en la subordinación y reorganización de los hechos desde una voluntad ideológica y política concreta, lo que necesita de un mecanismo de legitimación en el que se persigue naturalizar una epistemología basada en las emociones políticas, dado que las emociones y sentimientos son reales los hechos que los provocan, los desórdenes informativos, tienen que ser reales (Del-Fresno; Manfredi, 2018). Esto es, aquello que se siente -no sólo se siente- es, además, la verdad. Con la posverdad nos enfrentamos a una nueva forma de supremacía ideológica como herencia del totalitarismo -con rasgos evolutivos y adaptativos- en el seno de las democracias liberales en tiempos de Internet.

Los desórdenes desinformativos tienen ramificaciones que han permitido su eclosión actual:

a) la guerra contra la ciencia desde el ámbito corporativo;

b) la crisis de los medios de comunicación nacionales y locales post Internet;

c) el desarrollo de plataformas tecnológicas que han socializados la capacidad de publicar y distribuir contenidos a bajo coste;

d) la crisis de los expertos y los avances en psicología, para comprender las bases psicológicas, a través de diferentes sesgos cognitivos;

e) lo que ha provocado un cambio en la forma de entender el poder en el siglo XXI, como la capacidad de establecer las relaciones de definición (Beck, 2017) de la realidad misma.

\section{La duda como producto corporativo}

El ataque a la ciencia para la defensa de intereses corporativos es uno de los grandes afluentes intelectuales de los desórdenes desinformativos post Internet. Así, las redefiniciones de la realidad por medio de desórdenes informativos o, en su versión más básica, la fabricación de la duda

\section{A Frank Statement to Cigarette Smokers}

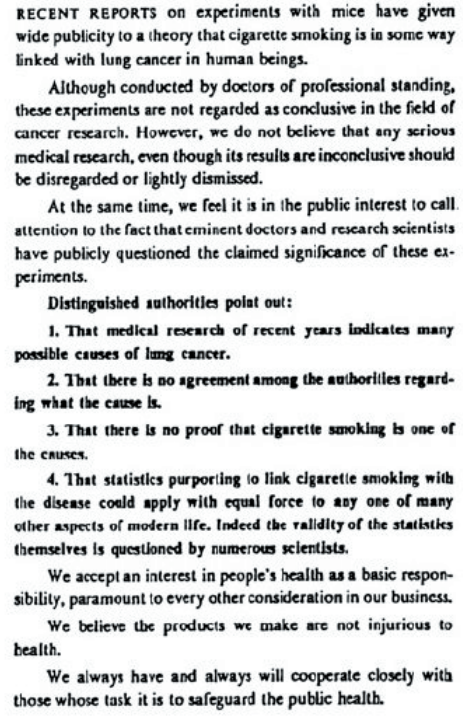

For more than 300 years tobacco has given solace, relaxation, and enjoyment to mankind. At one time or another during those years critics have held it responsible for practically every disease of the human body. One by one these charges have been abandoned for lack of eridence.

Regardless of the record of the past, the fact that cigarette smoking today should even be suspected as a cause of a serious smoking loday should even be suspected as a cause of a serio

Many people have asked us what we are doing to meet the answer:

1. We are pledging atd and assistance to the ressearch efliort into all phases of tobscero use and healith. This joint financisl sid will of course be in addition to what is alretdy being costribeted by malivideal companies.

2. For tha parpose we are cetablisting a potnt industry growp known as TOBACCO INDUSTRY RESEARCH COMMITTEE.

3. In charge of the research netivities of the Conumiltee mill be : sclentist of animpeachable Integrity and matlonal repule. In ested in the charelle as Adrisory Board of scientisis disinterfrom medicise, scrience, and education will be invited to merre

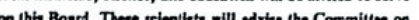
its research activitles.

This statement is being issued because we believe the people are entitled to know where we stand on this matter and what we intend to do about it.

\section{TOBACGO INDUSTRY RESEARCH COMMITTEE}
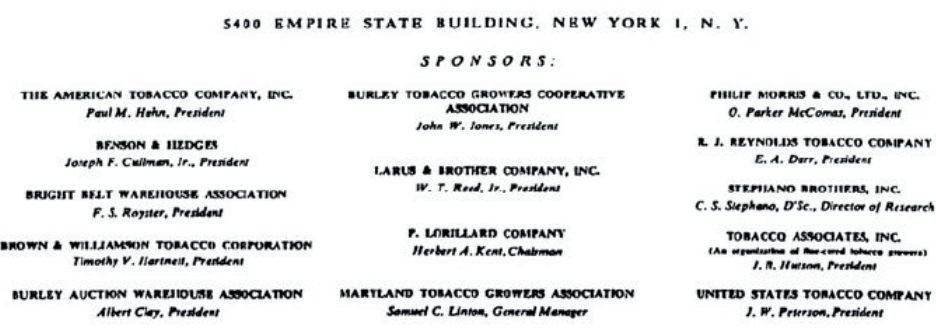

Figura 1. Campaña de publicidad de las tabacaleras en 1954. public's concern aroused by the recent reports. Here is the 
alrededor de las evidencias es algo que se produce a la luz pública a través de mecanismos bien conocidos (lobby, relaciones públicas, publicidad, financiación selectiva, propiedad mediática, etc.). Desde la segunda mitad del siglo XX tras la emergencia de descubrimientos científicos de gran calado, diferentes organizaciones corporativas desencadenaron campañas sistemáticas de desprestigio, cuestionamiento y pseudo debates con el objetivo de cuestionar y fabricar la duda entre la opinión pública contra las evidencias científicas disponibles. El objetivo en la lucha corporativa sistemática contra la ciencia y sus evidencias es evitar por completo o posponer toda toma de decisiones que pueda afectar de forma negativa a sus intereses. Esta lucha contra la ciencia a la luz del día se produce porque para sus promotores los costes no existen o bien, tras un cálculo de coste-utilidad, la promoción intencional de desórdenes informativos es económicamente rentable.

Un caso paradigmático es el de la industria tabacalera, que desde los años 50 del siglo XX primero negó y, luego, desinformó tras publicarse las primeras evidencias que relacionaban el consumo de tabaco y cáncer de pulmón (Dool; Hill, 1950; Norr, 1952). La reacción corporativa supuso un hito en la historia de la publicidad cuando en 1954 se financió una campaña de publicidad con el título "A frank statement to cigarette smokers" (figura 1). Se publicaron anuncios a página completa, de manera simultánea en más de 440 medios de impresos de EUA, que cuestionaban las evidencias científicas firmado por el Tobacco Industry Research Committee (imagen 1) creando la apariencia de que, en realidad, había una controversia científica. Esto es, declarando la guerra contra la ciencia con la apariencia de ciencia alrededor como cobertura para una campaña desinformativa. Esa guerra se extendió durante décadas hasta que las principales empresas fabricantes fueron declaradas culpables, más de medio siglo después, de "conspirar para negar, distorsionar y minimizar los riesgos de fumar cigarrillos para el público" (Smith et al., 2011, p. 690).

Algo muy similar sucedió con el descubrimiento de Rowland y Molina (1974) de la pérdida del ozono (ozone depletion) en altas capas de la atmósfera causada por las moléculas tipo CFCs -creadas en 1929 y presentes en multitud de utensilios y aparatos cotidianos- que estaban degradando la capa de ozono, lo que podía ocasionar graves daños para la vida en el planeta (figura 2). Al mismo tiempo que se consolidaba el consenso científico alrededor del descubrimiento de Rowland y Molina se inició la controversia negacionista promovida, en este caso, por la industria química que logró posponer más de una década la toma decisiones para detener su producción. Sólo cuando Farman, Gardiner y Shanklin (1985) mostraron la urgencia del problema y los efectos catastróficos de los CFCs la desinformación corporativa no pudo posponer la toma de decisiones para detener la producción de CFCs. Lo mismo, sucedió cuando Likens y Bormann (1974) publicaron sus investigaciones sobre los efectos irreversibles de la lluvia ácida en los ecosistemas debido a la contaminación y a otros problemas ecológicos (figura 3). También está documentado como grupos de corporaciones han fabricado y promovido controversias contra las evidencias científicas

"incluyendo la del tabaco, azúcar, maíz, salud, energía, pesticidas, armas, alcohol y muchas otras [...] orientadas a la manipulación de la investigación científica, legislación, discurso político y la comprensión pública” (Oreskes; Conway, 2010, p. 98)

o la farmacéutica de marcas, en España, desinformando sobre los medicamentos genéricos (Del-Fresno; López-Peláez, 2014), etc. En esta casuística de desinformación corporativa sistemática (Rabin-Havt, 2016) la tendencia apunta a que la ciencia se ve envuelta con lo comercial y la política lo que lleva a que muchos problemas, donde los científicos deberían ser los interlocutores válidos, acaben convirtiéndose en partisanismo ideológico, como sucede con el cambio climático (Mooney, 2012; 2005) y su negacionismo o la teoría evolucionista enfrentada por el creacionismo (Caudill, 2013).

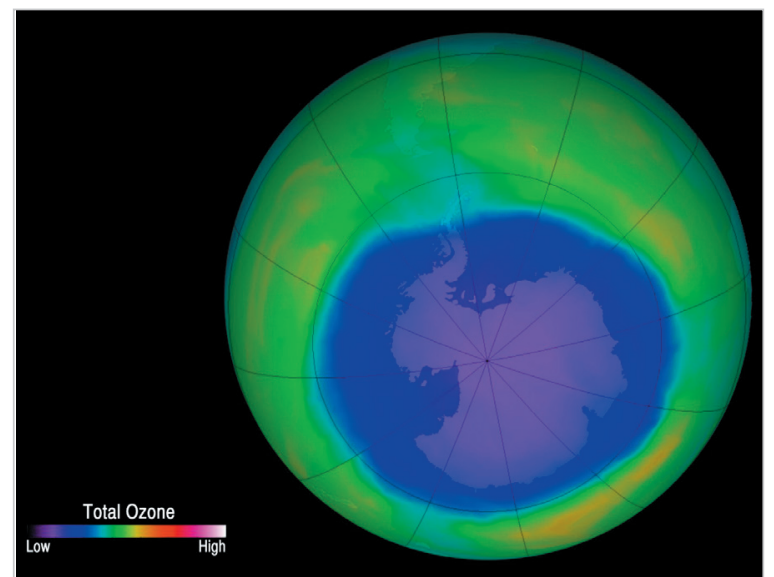

Figura 2. Concentraciones de ozono sobre la Antártida, 2 de octubre de 2015. Fuente: NASA / Goddard Space Flight Center

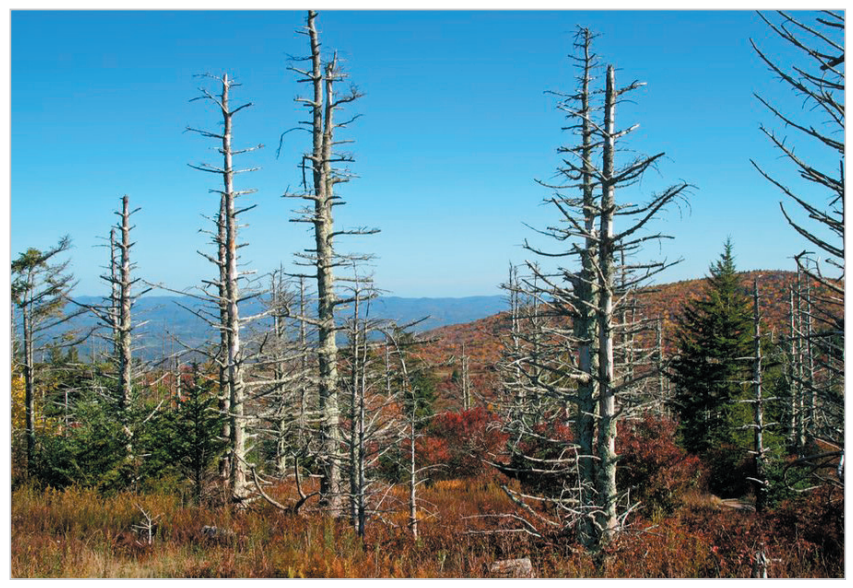

Figura 3. Efectos de la lluvia ácida

https://www.worldatlas.com/articles/how-is-acid-rain-formed.html 
Se ha demostrado cómo la desinformación corporativa financia su propia ciencia sesgada (biased production) distribuyéndola en los medios de comunicación o promocionando de forma selectiva sólo el conocimiento científico útil para los intereses corporativos o seleccionando aquellos datos espurios que vayan contra el consenso científico. La identificación de estas estrategias de lucha contra la ciencia muestran la existencia de
Los desórdenes informativos, acelerados por la innovación tecnológica, desde su origen corporativo han ido mutando hasta convertirse en un aspecto vertebral de la ideología política

"poderosas fuerzas en el mundo cuyos intereses dependen de la opinión pública [...] para promover sus propias agendas" (O'Connor; Weatherall, 2019, p. 92).

La dificultad estriba en mantener la legitimidad y autoridad social de la ciencia a pesar de que los patrones en la batalla corporativa contra la ciencia puedan ser identificados con claridad:

1) negar de forma activa las verdades fácticas por medio del cuestionamiento directo de las evidencias, acusándolas de insuficientes, creando la apariencia de incertidumbre con el objetivo de cuestionar o negar la simple existencia del problema identificado;

2) generar la alarma social de que actuar con insuficientes evidencias o de forma temprana contra el problema ya identificado, tendrá consecuencias negativas económicas y sociales;

3) financiar la investigación propia para que produzca evidencias sesgadas o a científicos que aporten resultados amistosos para la industria;

4) invertir la carga de la prueba hacia los científicos, creando falsas controversias públicas y exigiendo, en bucle, más evidencias para así ralentizar la toma decisiones;

5) sesgar la distribución de información de forma intencional de los propios resultados científicos y promoviendo los propios y polémicos para alimentar la falsa controversia;

6) erosionar el consenso científico, fabricar y socializar la duda para extender la percepción de riesgo y la persuasión emocional entre las audiencias donde el papel, de forma intencional o no, de los medios de comunicación profesionales y de las plataformas de Internet, en las últimas décadas, es crítico;

El impacto social de los desórdenes informativos no sería de tal magnitud si no fuese, además, por la crisis de los medios impresos de comunicación tras la emergencia de Internet

7) instrumentalizar el propio método científico para cuestionarlo -ya que la historia de la ciencia ha demostrado que la ciencia normal puede estar equivocada (Khun, 1962) al ser una construcción cultural y que todo conocimiento es válido hasta que es falseado-.

La socialización intencional del negacionismo científico y la desinformación sobre las evidencias fácticas acaba por desplazar el foco de atención del problema científico a la narrativa de la pseudo controversia en el debate público. Lo que supone en la práctica la capacidad de ejercer el poder de redefinición de la realidad fáctica por parte de los promotores de la desinformación.

El potencial catastrófico (Virilio, 1995) de los desórdenes informativos, acelerados por la innovación tecnológica con origen corporativo es que han mutado hasta convertirse en un aspecto vertebral de la ideología política. Y, por tanto, amplifican aún más sus consecuencias negativas a través de gobernanzas sesgadas que ignoran o posponen la toma de decisiones con graves consecuencias para la vida y la convivencia en el planeta, como sucede con el cambio climático. Este es el contexto que hace que temas cruciales globales, a pesar de estar dominados por un alto consenso científico como el cambio climático (Hoffman, 2012) o el creacionismo (figura 4) y su diseño inteligente (Caudill, 2013) han acabado por convertirse en guerras culturales que polarizan a las sociedades moral, social y políticamente. Y, en definitiva, crean grandes grupos sociales estables insensibles a las evidencias científicas.

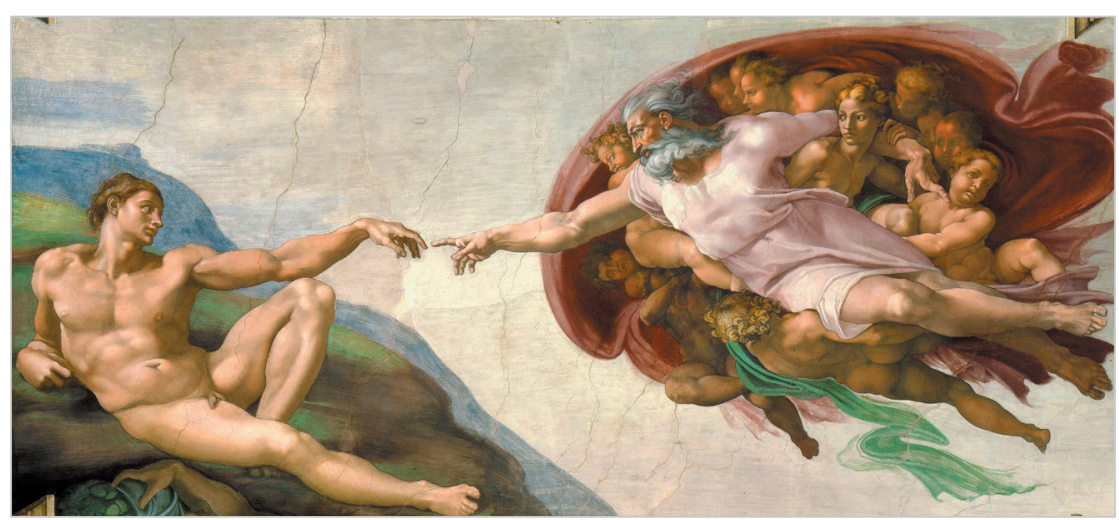

Figura 4. La creación de Adán (1510), fresco de Miguel Ángel (1475-1564) expuesto en la Capilla Sixtina del Vaticano, expresión gráfica del Creacionismo. 


\section{La crisis de los medios de comunicación con Internet}

El impacto social de los desórdenes informativos no sería de tal magnitud si no fuese, además, por la crisis de los medios impresos de comunicación tras la emergencia de Internet, que provocó que su modelo de negocio, basado en la publicidad, se haya desmoronado arrastrando al modelo editorial. El trasvase de inversión publicitaria hacia las grandes plataformas online, capaces de ofrecer segmentaciones de audiencias, precios y eficacia inigualables, arrastró a esos medios a un bucle vicioso de recortes en recursos humanos, el sacrificio de la calidad y rigor, la batalla por el clickbait, la pérdida de lectores reapropiados por la agregación y gestión algorítmica de noticias desde Facebook, Google y otras plataformas (Wills; Reeves, 2009; Del-Fresno; Daly, 2019). Plataformas que se han impuesto como intermediarios necesarios y en las cuales, de forma paradójica, esos lectores por millones consumen los contenidos de los medios profesionales (Del-Fresno, 2017). Además, los promotores de desórdenes informativos, en especial los más ideológicos, han creado sus propios canales lo que ha facilitado la emergencia y eclosión de pseudomedios, que con presentaciones profesionales y apariencia de legítimos, han extinguido la frontera entre información, opinión e ideología.

Pero otra causa de los desórdenes informativos se remonta al tiempo en el que los medios de comunicación dejaron de perseguir la información basada en verdades fácticas a favor de dar satisfacción a una objetividad o equilibrio de las diferentes versiones de la realidad, en muchos casos contrapuestas por completo. Al renunciar a la búsqueda y presentación de hechos verdaderos contrastados los medios de comunicación profesionales llevaron -de forma progresiva buscando su propia imparcialidad o fairness- a las audiencias los debates de opiniones pero también los

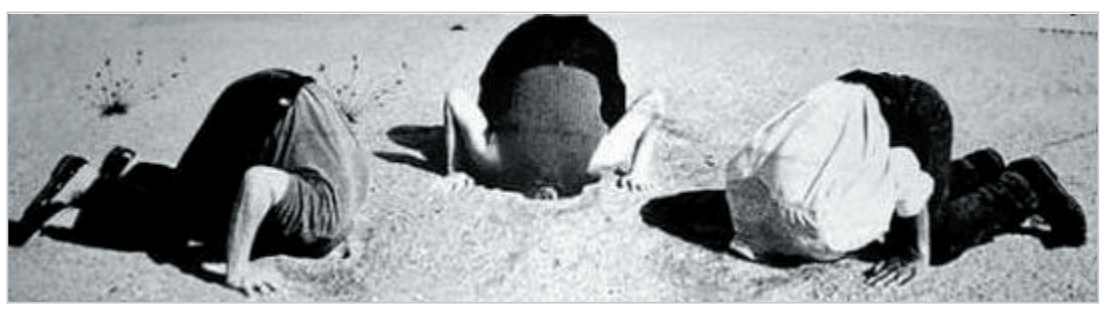

Figura 5. Negacionismo. Fotografía de fuente desconocida pseudo debates. La imparcialidad mediática degeneró en equilibrio como sesgo (Boykoof; Boykoff, 2004) cuando pasó de aplicarse de la presentación de opiniones divergentes a enfrentar los hechos científicos con el negacionismo intencional (figura 5). Esto es, presentando ante la opinión pública como interlocutores legítimos, como equivalentes y equipotentes, a científicos e ideólogos. Así, se acabó haciendo equivalente lo que no lo era y eliminando en la agenda pública la frontera entre verdades fácticas y desórdenes informativos. No obstante, el punto intermedio entre la verdad fáctica y los desórdenes informativos sigue siendo un desorden informativo.

Esto facilitó que cualquiera que reclamase ser un interlocutor legítimo pudiese reclamar su derecho a espacio y tiempo en los medios de comunicación para defender su posición. Y de no conseguirlo, acusar a medios de comunicación profesionales y periodistas de parcialidad o desacreditarlos por ello. Los medios profesionales de comunicación se convirtieron en una herramienta de los promotores de desórdenes informativos desde la esfera corporativa, propagandistas y promotores de teorías conspirativas o sin base científica.

Y para profundizar en la crisis de los expertos (Nichols, 2017), cientos de miles de individuos pueden convertirse en productores de desórdenes informativos utilizando plataformas tecnológicas ordinarias, que incluso con simples motivaciones económicas pueda tener consecuencias políticas globales como ocurrió en la carrera electoral entre Hillary Clinton y Donald Trump (Subramanian, 2017). La socialización tecnológica de la producción de contenidos tiene como consecuencia la creciente dificultad para establecer las líneas entre el periodismo profesional y la información partisana entre medios de comunicación profesionales y medios alternativos. Muchos de ellos eficaces a la hora de activar emocionalmente a grandes audiencias (Bakir; McStay, 2018) -como p.e. Infowars, Breitbart News y sus ramificaciones alrededor de la Alt-right (Willis, 2016; Ro-

Otra consecuencia de los desórdenes informativos es que crean grandes grupos sociales estables insensibles a las evidencias científicas, lo que ha facilitado la crisis de los expertos y generalizado una crisis epistémica

mano, 2017). El potencial catastrófico no sólo reside en la crisis de los medios profesionales de comunicación sino en que, además, no deja de crecer la dificultad para las audiencias a la hora de saber diferenciar a los meros opinantes (doxa) intencionales de los expertos (episteme). Otra consecuencia de esta crisis es que ha provocado, a su vez, una crisis de la autoridad epistémica.

\section{Bases psicológicas para la extensión de los desórdenes informativos}

La extensión de los desórdenes informativos ha tenido como factores de aceleración el conocimiento y uso intencional de los sesgos cognitivos, tanto de forma individual como colectiva, ya que son parte de nuestra herencia como especie. En las últimas décadas se han producido significativos avances en psicología (Khaneman, 2011) a partir de los estudios fundacionales (Tyersky; Khaneman, 1974; Kunda, 1990). Uno de los sesgos más asociados a los desórdenes informativos es el sesgo de confirmación o conformity bias (Asch, 1951) debido al cual los sujetos eligen alternativas contra las 
evidencias a causa de la presión social (figura 6). La necesidad de conformidad social y pertenencia a un grupo de referencia ejerce también una poderosa fuerza a la hora de percibir, explicar y comportarse en la vida cotidiana donde, de nuevo, la tecnología post Internet ha facilitado la creación de burbujas informativas, cámaras de eco (Anthony, 2016) y silos de información (Pariser, 2011). Herbert A. Simon, Premio Nobel de Economía, llamó racionalidad limitada (Simon, 1955; 1957; Neweel; Simon, 1972) a cómo debido a nuestras limitaciones cognitivas para profundizar en cada tema y problema nos lleva como individuos a elegir una solución satisfactoria, pero no necesariamente la mejor. Los desórdenes informativos soportados sobre sesgos cognitivos llevan a que todo evento pueda llegar a ser convertido, en la práctica, en eventos emocionales (Weeks, 2015). En especial, en relación con los eventos políticos donde la racionalidad y los mismos hechos tienden a estar comprometidos. Los desórdenes informativos soportados en sesgos cognitivos pueden llevar la construcción de una suerte de epistemología emocional (Del-Fresno; Manfredi, 2018) basada en que puesto que

"las emociones y los sentimientos son reales, se concluye que los objetivos también son reales y, por lo tanto, las emociones compartidas son importantes. Es decir, la emoción y los sentimientos se equiparan a la verdad y la legalidad. Así es como se fabrica la epistemología, y también la legalidad de la post-verdad" (Del-Fresno; Manfredi, 2018, p. 1232).

Las cascadas de información que facilita la tecnología permiten que los desórdenes informativos se extiendan entre grupos sin que se tengan en cuenta las evidencias que las refutan (p.e., la teoría de The Flat Earth) (figura 7).

Creencias que en la práctica no tienen consecuencias para sus promulgadores pero que sí las pueden tener para terceros cuando los desórdenes informativos incrementan los riesgos para la salud pública (Zika, Ébola, HIV, vacunas, etc.). La importancia de la difusión de los desórdenes en su intersección con las tecnologías post Internet ha demostrado ser crítica ya que se ha mostrado como su impacto, escala y velocidad (Vosoughi et al., 2018) no tiene precedentes históricos. Esto su-

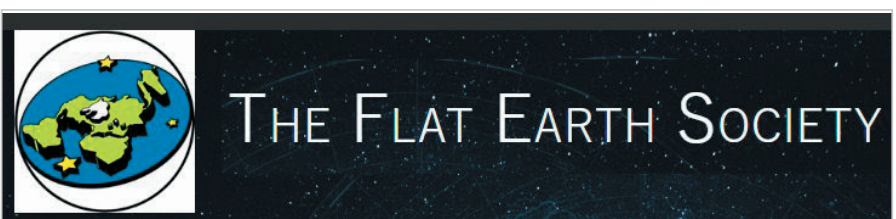

Figura 7. The Flat Earth Society - Index http://bit.ly/2Uh503g

The Flat Earth Wiki http://bit.ly/2Uc8X9D

pone efectos indeseados con un nuevo potencial catastrófico debido a que los productores de desórdenes informativos persiguen conseguir influencia social y modificar las percepciones, por tanto, influir en el comportamiento de individuos y sociedades. El riesgo asociado a las cascadas de desórdenes informativos a gran escala gracias, por ejemplo, a Twitter se ha demostrado que a diferencia de cascadas de información falsa las cascadas informativas con desinformación pueden impactar a grandes audiencias durante similares períodos de tiempo a la información verdadera (Vosoughi et al., 2018). Esto supone un cambio radical en la forma en que los desórdenes informativos impactan y la incapacidad práctica a la hora de pararlos o frenarlos.

En definitiva, los desórdenes informativos han encontrado en la psicología el caldo de cultivo y las condiciones de posibilidad idóneas

"para aquellos que tienen intereses en promover la tormenta perfecta para la explotación de nuestra ignorancia y sesgos cognitivos" (McIntyre, 2018, p. 114).

\section{Batalla por el poder de definición de lo real}

Los desórdenes informativos no suponen una posición filosófica o epistemológica contra la verdad fáctica sino la mera subordinación, intencional, de los hechos a un punto de vista económico o ideológico, lo que significa una voluntad de autoridad sobre la realidad, en la práctica, una voluntad de supremacía ideológica. Por tanto, la clave no residiría en qué significa la verdad sino en cómo y quién es capaz de establecer las condiciones de verosimilitud de la realidad. En palabras de Ulrick Beck se ha producido un desplazamiento desde

"foco del poder y de la dominación, en las relaciones de producción (en el sentido marxista) en el capitalismo global moderno, a las relaciones de poder de definición" (Beck, 2017, p. 115).

Ello lleva a la formulación de una teoría del poder: cómo y quién tiene la capacidad para definir la realidad y, por tanto, la agenda pública no sólo de forma local o regional sino global. 
Un ejemplo anticipatorio de este tipo de prácticas a la hora de imponer la definición de la realidad misma proviene del asesor político de G. W. Bush, Karl Rove cuando afirmó a un periodista de The New York Times:

"Ahora somos un imperio, y cuando actuamos, creamos nuestra propia realidad. Y mientras estudias esa realidad, cosa que lógicamente harás, nosotros actuaremos de nuevo, creando otras nuevas realidades" (Suskind, 2004).

Otro caso emblemático de voluntad de definir la realidad, a partir de desórdenes informativos, son los hechos alternativos o alternative facts que representan, acaso, la versión más cínica de los mismos. El concepto lo popularizó Kellyanne Conway, consejera de Donald Trump, enfrentada a la evidencia de que el Secretario de Prensa de la Casa Blanca había falseado la cifra de asistentes a la investidura presidencial de Donald Trump, como presidente de los Estados Unidos. Conway afirmó que la falsedad no era tal sino que se trataba de "alternative facts" (NBC News, 2016) (figura 8). La apelación al derecho de ofrecer hechos alternativos es una variación más de los desórdenes informativos puesto que no hay

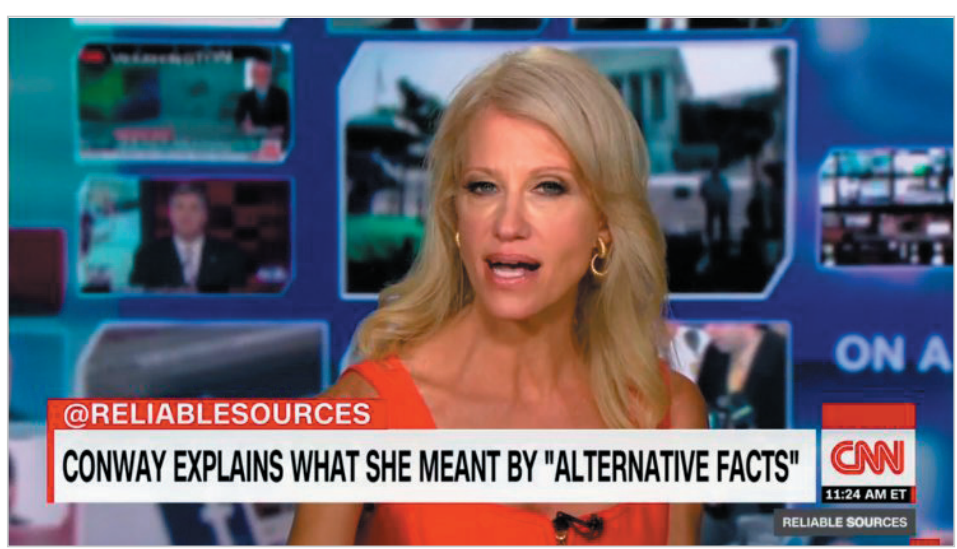

Figura 8. Crítica a los “hechos alternativos" de la consejera de Donald Trump desde la CNN

hechos alternativos sino voluntad de imponer ciertas percepciones de hechos, a costa de los hechos objetivos. En la práctica supone bien, una vuelta a una comprensión precientífica del mundo, o bien, la voluntad política de extender los desórdenes informativos como cinismo ideológico. Los hechos alternativos suponen un desafío último a las verdades fácticas. No obstante, los desórdenes informativos no tienen por qué haber culminado en el cinismo de los hechos alternativos.

Así, se evidencia como la intencionalidad de producir desórdenes informativos facilita que toda opinión o evento sea susceptible de ser elevado a verdad en la medida en que resulte útil a una cierta ideología, cosmovisión política o causa corporativa. Y, en definitiva, los desórdenes informativos, en especial la desinformación, no se basan para su aceptación social en la coacción propagandística sino en la libertad de los ciudadanos para elegirlos de forma voluntaria (Del-Fresno; Manfredi, 2018).

No obstante, cabe destacar que la desinformación es un concepto más multifacético y complejo que la mentira, lo inexacto o lo incompleto (Karlova; Lee, 2011) puesto que al estar asociada a formas de intencionalidad, contexto y significado tiene, incluso en su deformación, siempre un carácter informativo (Buckland, 1991). El riesgo asociado

Por evidentes que sean los patrones de cómo funcionan los desórdenes informativos sigue siendo muy significativa la dificultad para diferenciar las verdades fácticas de las ficciones

a la desinformación ha sido identificado como una de las amenazas emergentes para las sociedades por el World Economic Forum (Howell, 2013). La desinformación, incluso siendo falsa -en parte o por completo- es una especie de información puesto que "informar no requiere verdad, y la información no necesita ser verdad" (Fox, 1983, p. 193).

De igual manera que el poder no puede reducirse a la violencia física, la complejidad de la desinformación no permite reducir su comprensión y análisis a las fake news, lo inexacto o lo incompleto, sino que como desorden informativo la desinformación sigue siendo informativa. Y, aún más importante, su potencial catastrófico reside en que su intersección con la política y debido a su carácter performativo (Austin, 1975 [1962]) se persiguen objetivos que tienen como objetivo transformar la realidad (Del-Fresno; Manfredi, 2018).

\section{Conclusiones}

De forma colectiva, como sociedades, somos eficaces abordando y superando debates filosóficos, pero mucho menos en lo que se refiere a protegernos de las consecuencias de los desórdenes informativos y las irracionalidades cognitivas. La escala, alcance y velocidad de los desórdenes informativos, interrelacionados entre sí, en intersección con las nuevas tecnologías post Internet está transformado la naturaleza misma de la comunicación interpersonal colectiva, en especial la relacionada con la política.

Por evidentes que sean los patrones de cómo funcionan los desórdenes informativos sigue siendo muy significativa la dificultad para diferenciar las verdades fácticas de las ficciones. El resultado es que las audiencias siguen confusas y el potencial catastrófico de las consecuencias crece. Al mismo tiempo que estamos sobreexpuestos a diario a enormes cantidades de impactos reclamando nuestra atención, moldeando nuestras percepciones y afectando a nuestra comprensión de la realidad y comportamiento estaríamos alcanzando, de forma simultánea, niveles críticos en la dificultad para identificar la información fiable de la espuria o aquella sesgada de forma intencional.

Cuanto más difícil se hace para los ciudadanos separar la información fiable y las evidencias científicas de los desórdenes informativos más probable es que la construcción de la opinión pública, y las consecuencias que pueda tener en la agenda colectiva, se base en hechos espurios. 
La evolución previsible apunta hacia una mayor complejidad y entropía social. Por un lado, la evolución tecnológica -con la ayuda de la inteligencia artificial- no dejará de ser utilizada para producir desórdenes informativos de mayor complejidad y sofisticación como los deepfakes. Por otro, es previsible que la dificultad para diferenciar desórdenes informativos intencionales de la información veraz, soportada en un conocimiento más exacto de los sesgos cognitivos, no deje de crecer por lo que se hace más urgente el poder identificar y penalizar con rapidez a los responsables de desórdenes desinformativos, defender el consenso entre expertos y socializar el conocimiento científico.

La verdad fáctica es una empresa colectiva y su vigencia y extensión demuestran el éxito de esa empresa en nuestra historia como especie. De igual forma es necesario recuperar el reconocimiento y respeto social de los científicos, como fuentes de autoridad y credibilidad, frente a los propagandistas, demagogos y desinformadores impulsados por las fuerzas del interés particular y totalitario. La ciencia sigue siendo la la respuesta necesaria como método y base para sostener las necesidades de las sociedades democráticas.

\section{Referencias bibliográficas}

Alcorn, Gay (2014). "Facts are futile in an era of post-truth politics". The age, 28 February. http://bit.ly/2iKT7BI

Anthony, Andrew (2016). "Inside the hate-filled echo chamber of racism and conspiracy theories". The guardian, 18 December. $h$ ttps://goo.gl/kvagRP

Arendt, Hannah (1951). The origins of Totalitarianism. New York: Schocken [Elemente und Ursprünge totaler Herrschaft] (revised ed.). Houghton Mifflin Harcourt. ISBN: 9780547543154

Asch, Solomon E. (1951). "Effects of group pressure upon the modification and distortion of judgments". In: H. Guetzkow (ed.). Groups, leadership and men: research in human relations (pp. 177-190). Oxford, England: Carnegie Press.

Austin, J.L. (1975[1962]). How to do things with words. Oxford: Oxford University Press. https://goo.gl/kvagRP

Bakir, Vian; McStay, Andrew (2018). "Fake news and the economy of emotions", Digital journalism, v. 6, n. 2, pp. $154-175$. https://doi.org/10.1080/21670811.2017.1345645

Beck, Ulrick (2016). The metamorphosis of the world: How climate change is transforming our concept of the world. Cambridge: Polity press. ISBN: 9780745690223

Bikhchandani, Sushil; Hirshleifer, David; Welch, Ivo (1992). "A theory of fads, fashion, custom, and cultural change in informational cascades". Journal of political economy, v. 100, n. 5, pp. 992-1026.

https://doi.org/10.1086/261849

Boykoff, Maxwell T.; Boykoff, Jules M. (2004). "Balance as bias: global warming and the US prestige press". Global environmental change, v. 14, n. 2, pp. 125-136.

https://doi.org/10.1016/j.gloenvcha.2003.10.001

Buckland, Michael (1991). "Information as thing". Journal of the American Society for Information Science, v. 42, n. 5, pp. $351-60$. https://doi.org/10.1002/(SICI)1097-4571(199106)42:5<351::AID-ASI5>3.0.CO;2-3

Caudill, Edward (2013). Intelligently designed: How creationists built the campaign against evolution. University of Illinois Press. ISBN: 9780252038013

Del-Fresno, Miguel (2017). "We the new media: The disruption of social media in interpersonal and collective communication”. In: Cabrera, Margarita; Lloret, Nuria (2017). Digital tools for academic branding and self-promotion. Cincinnati: IGI-Books, pp. 11-30. ISBN: 9781522509172

https://doi.org/10.4018/978-1-5225-0917-2

Del-Fresno, Miguel; Daly, Alan-James (2019). "Limits for the political communication through large online platforms: from The caste to The plot". Revista española de investigaciones sociológicas, n. 165, pp. 23-42.

https://goo.gl/6p7Qpa

Del-Fresno, Miguel; López-Peláez, Antonio (2014). "Social work and netnography: The case of Spain and generic drugs". Qualitative social work, v. 13, n. 1, pp. 85-107. https://doi.org/10.1177/1473325013507736

Del-Fresno-García, Miguel; Manfredi-Sánchez, Juan-Luis (2018). “Politics, hackers and partisan networking. Misinformation, national utility and free election in the Catalan independence movement". El profesional de la información, v. 27, n. 6, pp. 1225-1238.

https://doi.org/10.3145/epi.2018.nov.06

Del-Vicario, Michela; Bessi, Alessandro; Zollo, Fabiana; Petroni, Fabio; Scala, Antonio; Caldarelli, Guido; Stanley, H. Eugene; Quattrociocchi, Walter (2016). "The spreading of misinformation online". Proceedings of the National Academy 
of Sciences of the United States of America (PNAS), v. 113, n. 3, pp. 554-559.

https://doi.org/10.1073/pnas.1517441113

Doll, Richard; Hill, A. Bradford (1950). "Smoking and carcinoma of the lung". British medical journal, v. 2, pp. 739-748. https://doi.org/10.1136/bmj.2.4682.739

Drezner, Daniel (2016). "Why the post-truth political era might be around for a while". The Washington Post, 16 June. http://wapo.st/2iLqe7Z

European Commission (2018). "A multi-dimensional approach to disinformation. Report of the independent High Level Group on Fake News and Online Disinformation". Directorate-General for Communication Networks, Content and Technology. http://bit.ly/2Ponxc1

Farman, Joseph-Charles; Gardiner, Brian G.; Shanklin, Jonathan D. (1985). "Large losses of total ozone in Antarctica reveal seasonal $\mathrm{ClO}_{\mathrm{x}} / \mathrm{NO}_{\mathrm{x}}$ interaction". Nature, n. 315, pp. 207-210.

https://doi.org/10.1038/315207a0

Flood, Alison (2016). "'Post-truth' named word of the year by Oxford dictionaries”. The guardian, 15 November. http://bit.ly/2iKOUOu

Fox, Christopher (1983). Information and misinformation: An investigation of the notions of information, misinformation, informing, and misinforming. Westport, CT: Greenwood. ISBN: 9780313239281

Frankfurt, Harry G. (2005). On bullshit. Princeton, NJ: Princeton University Press.

Freedland, Jonathan (2016). "Post-truth politicians such as Donald Trump and Boris Johnson are no joke”. The guardian, 13 May. http://bit.ly/2iKKMxr

Hernon, Peter (1995). "Disinformation and misinformation through the internet: Findings of an exploratory study". Government information quarterly, v. 12, n. 2, pp. 133-139.

https://doi.org/10.1016/0740-624X(95)90052-7

Hoffman, Andrew J. (2012). “Climate science as culture war”. Stanford social innovation Review, v. 10, n. 4, pp. 30-37.

Howell, Lee (2013). "Digital wildfires in a hyperconnected world". WEF report 2013.

http://bit.ly/2hpiDxa

Karlova, Natascha; Lee, Jin-Ha (2011). "Notes from the underground city of disinformation: A conceptual investigation". In: Proceedings of the Asist, October, 9-13, New Orleans, LA.

http://bit.ly/2x4KDNp

Khaneman, Daniel (2011). Thinking fast and slow. New York: Farrar, Strauss \& Giroux.

Koyré, Alexandre (1945) “The political function of the modern lie”, Contemporary Jewish record, v. 8, n. 3, pp. 290-300.

Kuhn, Thomas S. (1971). La estructura de las revoluciones científicas. México: Fondo de Cultura Económica.

Kunda, Ziva (1990). "The case for motivated reasoning". Psychological bulletin, v. 108, n. 3, pp. 480-498.

https://doi.org/10.1037/0033-2909.108.3.480

Lazer, David M. J.; Baum, Mathew A.; Benkler, Yochai; Berinsky, Adam J.; Greenhill, Kelly M.; Menczer, Filippo; Metzger, Miriam J.; Nyhan, Brendan; Pennycook, Gordon; Rothschild, David; Schudson, Michael; Sloman, Steven A.; Sunstein, Cass R.; Thorson, Emily A.; Watts, Duncan J.; Zittrain, Jonathan L. (2018). "The science of fake news". Science, v. 359, n. 6380, pp. 1094-1096.

https://doi.org/10.1126/science.aao2998

Likens, Gene E.; Bormann, F. Herbert (1974) “Acid rain: a serious regional environmental problem”. Science, v. 184, n. 4142, pp. 1176-1179.

https://doi.org/10.1126/science.184.4142.1176

McIntyre, Lee (2018). Posverdad. Madrid: Cátedra. ISBN: 9788437638690

Molina, Mario J.; Rowland, F. Sherwood (1974). "Stratospheric sink for chlorofluoromethanes: Chlorine atom-catalysed destruction of ozone". Nature, n. 249, pp. 810-812.

https://www.nature.com/articles/249810a0

Mooney, Chris (2005). The Republican war on science. Nuew York: Basic Books. ISBN: 9780465046768

Mooney, Chris (2012). The Republican brain: the science of why they deny science - and reality. Hoboken, NJ: Wiley. ISBN: 9781118094518

NBC News (2017). "Kellyanne Conway: Press secretary Sean Spicer gave 'alternative facts'”, NBC News, 22 Jan., YouTube. http://bit.ly/2uziMUy 
Newell, Allen; Simon, Herbert A. (1972). Human problem solving. New York: Prentice Hall. ISBN: 9780134454030

Nichols, Tom (2017). The death of expertise: the campaign against established knowledge and why it matters. Oxford: Oxford University Press. ISBN: 9780190469412

Norr, Roy (1952). "Cancer by the carton". Reader's digest, December. https://csts.ua.edu/files/2019/01/1952-12-Readers-Digest-Cancer-by-the-Carton.pdf

O'Connor, Cailin; Weatherall; James O. (2019). The misinformation age: How false beliefs spread. Yale University Press ISBN: 9780300234015

Oreskes, Naomi; Conway, Erik M. (2012). Merchants of doubt: how a handful of scientists obscured the truth on issues from tobacco smoke to global warming. London: Bloomsbury.

Oxford University Press (2016). "Word of the Year 2016 is...". Oxford dictionaries. http://bit.ly/2BzsaJf

Pariser, Eli (2011). The filter bubble: how the new personalized web is changing what we read and how we think. Penguin Books. ISBN: 0143121235

Rabin-Havt, Ari (2016). Lies, incorporated: The world of post-truth politics. New York: Anchor Books. ISBN: 9780 307279590

Romano, Aja (2017). "The alt-right drove feminist writer Lindy West off Twitter. That has real-world political implications". Vox, 6 January. http://bit.ly/2i4FJXO

Simon, Herbert A. (1955). "A behavioral model of rational choice”. Quarterly journal of economics, v. 69, n. 1, pp. $99-118$. https://doi.org/10.2307/1884852

Simon, Herbert A. (1957). Models of man, social and rational: Mathematical essays on rational human behavior in a social setting. New York: John Wiley and Sons.

Smith, Philip; Bansal-Travers, Maansi; O’Connor, Richard; Brown, Anthony; Banthin, Chris; Guardino-Colket, Sara; Cummings, Michael (2011). "Correcting over 50 years of tobacco industry misinformation", American journal of preventive medicine, v. 40, n. 6, 2011, pp. 690-698. https://doi.org/10.1016/j.amepre.2011.01.020

Subramanian, Samanth (2017). "Inside the Macedonian fake-news complex". Wired, 17 Febr. http://bit.ly/2IxQnrf

Suskind, Ron (2004). "Faith, certainty and the presidency of George W. Bush". The New York Times, 17 Oct. https://nyti.ms/2uzDofE

Tversky, Amos; Kahneman, Daniel (1974) “Judgment under uncertainty: heuristics and biases”. Science, v. 185, n. 4157, pp. 1124-1131.

http://www.jstor.org/stable/1738360

Virilio, Paul (1991). The aesthetics of disappearance. New York: Semiotext(e). ISBN: 9781570270413

Virilio, Paul (1995). Speed and information: Cyberspace alarm!

http://www.ctheory.net/articles.aspx?id=72

Vosoughi, Soroush; Roy, Deb; Aral, Sinan (2018). "The spread of true and false news online". Science, v. 359, n. 6380, pp. 1146-1151. https://doi.org/10.1126/science.aap9559

Wardle, Claire; Hossein, Derakhshan (2017). Information disorder: Toward an interdisciplinary framework for research and policymaking. Report to the Council of Europe.

http://bit.ly/2XsvEs7

Weeks, Brian E. (2015). "Emotions, partisanship, and misperceptions: How anger and anxiety moderate the effect of partisan bias on susceptibility to political misinformation". Journal of communication, n. 65, n. 4, pp. 699-719. https://doi.org/10.1111/jcom.12164

Willis, Oliver (2016). "What is the 'Alt-Right'? A guide to the white nationalist movement now leading conservative media". Mediamatters.org, August $25^{\text {th }}$.

http://bit.ly/2zsWXty

Wills, Davis; Reeves, Stuart (2009). "Facebook as a political weapon: Information in social networks". British politics, v. 4 n. 2, pp. 265-281.

https://doi.org/10.1057/bp.2009.3 